PALEO

Revue d'archéologie préhistorique

$13 \mid 2001$

Varia

\title{
L'aven des Fourches II (Sault, Vaucluse) : les derniers chevaux sauvages en Provence
}

Last wild horses in Provence: the sinkholes of Les Fourches II (Sault, Vaucluse)

Jean-Philip Brugal, Jacques Buisson-Catil et Daniel Helmer

\section{OpenEdition}

Journals

Édition électronique

URL : http://journals.openedition.org/paleo/974

DOI : $10.4000 /$ paleo.974

ISSN : 2101-0420

Éditeur

SAMRA

Édition imprimée

Date de publication : 14 décembre 2001

Pagination : 73-88

ISSN : $1145-3370$

\section{Référence électronique}

Jean-Philip Brugal, Jacques Buisson-Catil et Daniel Helmer, «L'aven des Fourches II (Sault, Vaucluse) : les derniers chevaux sauvages en Provence », PALEO [En ligne], 13 | 2001, mis en ligne le 26 mai 2010, consulté le 07 juillet 2020. URL : http://journals.openedition.org/paleo/974 ; DOI : https://doi.org/ 10.4000/paleo.974

Ce document a été généré automatiquement le 7 juillet 2020

\section{cc) (i) (9)}

PALEO est mis à disposition selon les termes de la licence Creative Commons Attribution - Pas d'Utilisation Commerciale - Pas de Modification 4.0 International. 


\title{
L'aven des Fourches II (Sault, Vaucluse) : les derniers chevaux sauvages en Provence
}

\author{
Last wild horses in Provence: the sinkholes of Les Fourches II (Sault, Vaucluse)
}

Jean-Philip Brugal, Jacques Buisson-Catil et Daniel Helmer

Nous tenons à remercier ici Monsieur René Liardet pour son aide et l'accueil chaleureux qu'il nous a réservé.

\section{Introduction}

1 Le gisement paléontologique des Fourches II a été découvert fortuitement en 1990 par R. Liardet $t^{4}$, le propriétaire du terrain. Très exposée aux déprédations et aux fouilles clandestines favorisées par son isolement dans une zone fréquemment parcourue par des spéléologues amateurs et des randonneurs, la cavité fit l'objet au mois de mai 1991 d'une petite opération de sondage, conduite par le Service d'Archéologie du Conseil général de Vaucluse, ayant pour objectifs de vérifier la présence éventuelle de vestiges archéologiques et de prendre les dispositions nécessaires à sa protection.

\section{Le cadre géographique et géologique}

\section{Situation}

2 L'aven des Fourches II est situé sur la commune de Sault, à environ $2 \mathrm{~km}$ au nord-ouest du village et à $70 \mathrm{~km}$ au nord-est d'Avignon (fig. 1). Creusé dans un des reliefs du Crétacé inférieur qui couvrent près des deux tiers de la superficie du département de Vaucluse, il s'ouvre sur un plateau fortement karstifié dans une zone, entre la chaîne septentrionale du Mont Ventoux au nord et la chaîne du Luberon au sud, qui englobe le versant sud du "Géant de Provence" et les monts de Vaucluse (fig. 2). 
Figure 1 : Situation de l'aven des Fourches II.

Figure 1: Geographic location of the sinkholes of Les Fourches II.

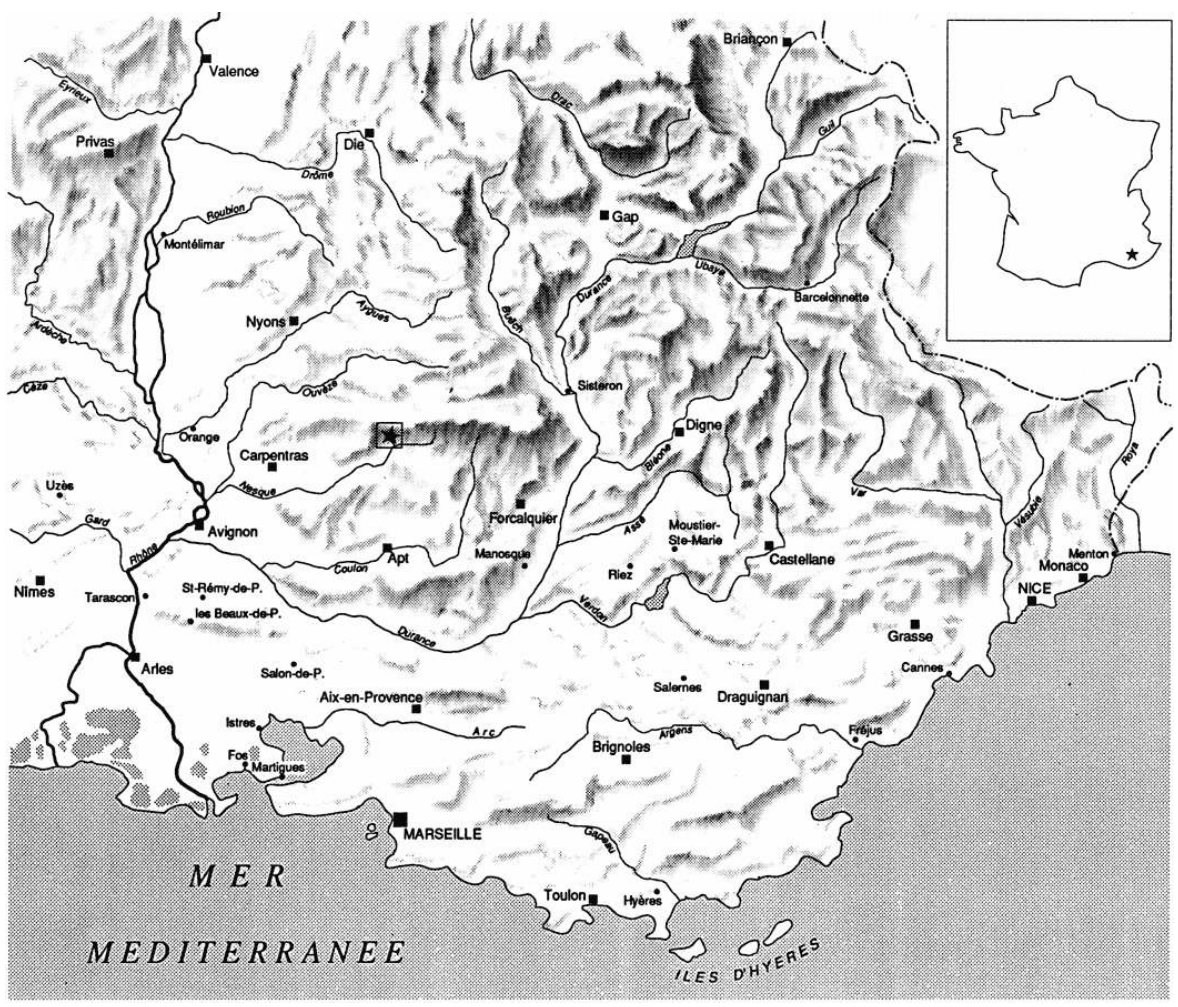

Figure 2 : Carte géologique simplifiée et principaux traits de fracturation (d'après J.-M. Puig, 1990, modifié).

Figure 2: Simplified geologic map and main faultings in north Provence (after Puig, 1990, modified).

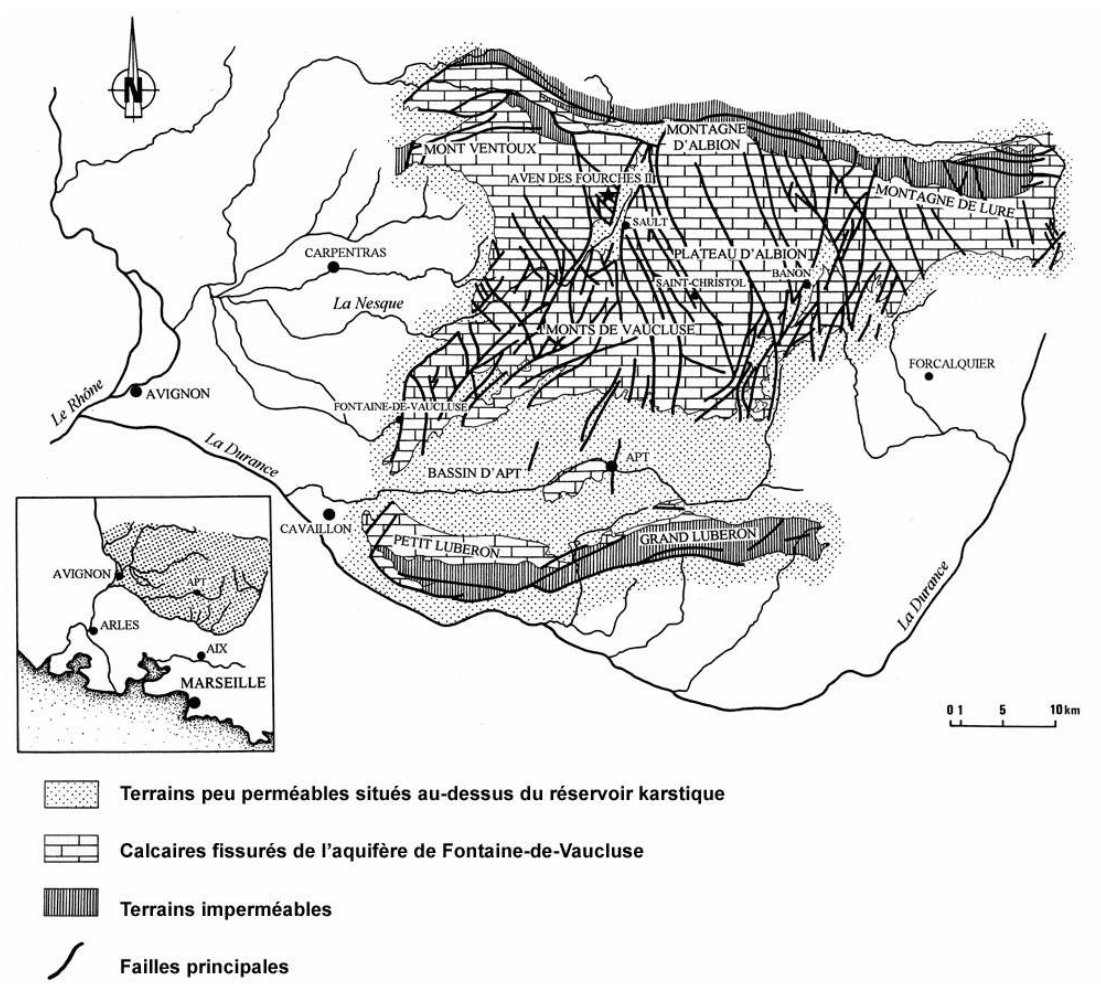


3 La diaclase s'ouvre à $795 \mathrm{~m}$ d'altitude, au milieu des buis, des chênes pubescents et des genévriers communs, dominant d'une centaine de mètres au sud le fossé de Sault arrosé par la Nesque encore pérenne dans cette partie de son cours et, à l'ouest, l'étroite combe du Fraysse qui la sépare du plateau des Molières.

4 A proximité immédiate de la cavité s'ouvre l'aven des Fourches I qui a livré, à la faveur d'opérations archéologiques d'envergure, de très nombreux vestiges attribuables au Chalcolithique et surtout à l'Age du Bronze final - Bronze D et Hallstatt A - (BuissonCatil 1993, 1996, Buisson-Catil et al. 1996-1997). Toujours dans ce secteur s'ouvre l'aven du Quartier du Ventoux 2, bien connu des spéléologues, où après un petit ressaut de 2,50 mètres, on aboutit dans une galerie d'assez belles dimensions pour le plateau. C'est en fait l'ensemble de ce plateau qui est concerné par la karstification et on ne peut exclure l'existence de réseaux communiquants (fig. 3).

Figure 3 : Carte topographique du secteur sud du fossé de Sault et des plateaux bordiers.

Figure 3: Topography of south sector of Sault with plateaux and main sinkholes (1 to 9).

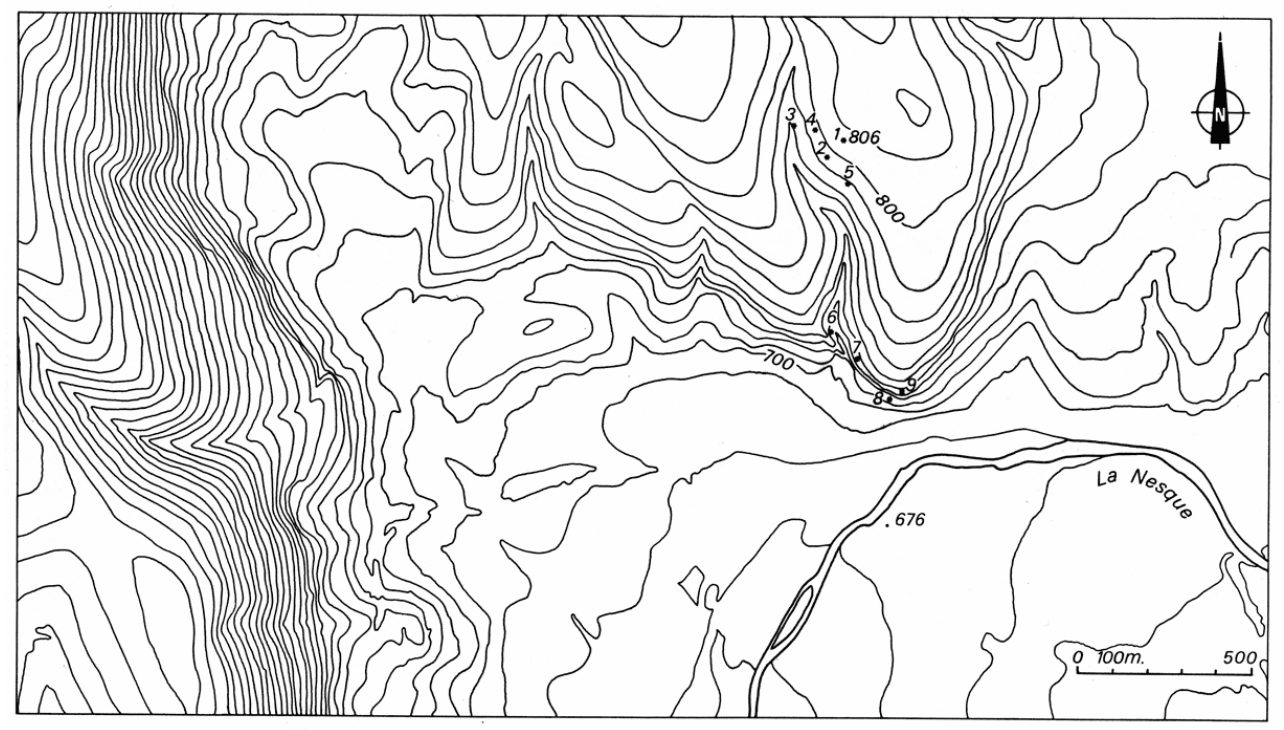

I: aven des Fourches I; 2 : aven des Fourches II; 3 : aven des Fourches III ; 4 : aven du quartier du Ventoux $2 ; 5$ : aven du quartier du Ventoux $1 ; 6$ : aven dou Cat $; 7$ : aven de la Loge $n^{\circ} 2 ; 9$ : aven de la Loge $n^{\circ} 1$

5 Si les formes souterraines de la karstification semblent ici moins nombreuses et moins spectaculaires que dans la partie extrême orientale des monts de Vaucluse, plateau de Saint-Christol et d'Albion (Gaubert et al. 1990, 1995 ; Gaubert 1993), il n'en demeure pas moins que le secteur qui nous intéresse se présente souvent sous l'aspect d'une région karstique typique, c'est-à-dire avec des surfaces lapiazées et nues (le sol étant constitué par la surface calcaire très ciselée et découpée par la dissolution) - karst thermique qui constitue le lapiaz stricto sensu. L'unité sous-jacente (karst de subsurface - conduits sous-cutanés), bien représentée, comprend tous les systèmes de fissures élargies ou non par la dissolution: cette action peut être augmentée dans certains secteurs par la fracturation tectonique (canevas tectonique local) en rapport avec les lithofaciès, comme en témoigne l'alignement préférentiel des dolines, des poljés et des avens le long des failles (Weydert 1990 ; Jorda 1993). L'épaisseur de cette unité varie de quelques mètres à quelques dizaines de mètres. La plupart du temps, la pénétration 
spéléologique de cette zone est très limitée; on n'en connait que les diaclases profondes et assez larges comme c'est le cas pour l'aven des Fourches II.

\section{Géologie et géomorphologie}

6 Pour bien comprendre la morphologie actuelle du fossé de Sault et de ses environs, il faut reprendre l'histoire géologique de la région depuis le début du Crétacé (Puig 1990). C'est en effet au Crétacé inférieur que se déposent, dans une mer profonde et subsidente, les sédiments calcaires qui, consolidés par les phénomènes diagénétiques, vont former ce que l'on a pu appeler le "panneau de couverture nord-provençal" (Villéger 1984): il s'agit d'une vaste dalle épaisse de plus de $1000 \mathrm{~m}$, en strates décimétriques et décamétriques à peine entrecoupées de rares lits marneux, et qui présente donc mécaniquement une grande rigidité. Après dépôt de ces sédiments calcaires, la mer devient plus profonde et reçoit les marnes gargasiennes. Puis, une phase tectonique correspondant à une compression nord-sud va amorcer des structures anticlinales et synclinales : du fait de la rigidité du panneau nord-provençal, ces plis d'orientation est-ouest ont un très grand rayon de courbure. Cette compression s'accompagne d'un exhaussement $d u$ fond marin et de l'émersion du sommet des anticlinaux. On pense que, dès cette phase, est exondée l'amorce des chaînes VentouxLure et Luberon et surtout d'un anticlinal au sud du Mont Ventoux, la structure de Saint-Gens (Rivier 1960).

7 Au milieu du Crétacé commence, avec une émersion générale de la région, une longue et intense période d'érosion des zones les plus hautes. Pour les calcaires massifs qui forment l'ossature des reliefs, cette érosion est essentiellement une karstification qui se traduit, en surface, par une ablation pouvant atteindre plusieurs centaines de mètres et, en profondeur, par le développement de réseaux de drainage souterrain qui se mettent en place à la faveur des failles et diaclases. Ces zones hautes sont restées soumises à l'érosion depuis cette époque, c'est-à-dire depuis 90 millions d'années.

8 Au début de l'oligocène, intervient une phase tectonique distensive avec un étirement orienté est-ouest. Certaines cassures antérieures rejouent en failles normales, principalement celles orientées NE-SO. Des compartiments entiers de la dalle calcaire s'affaissent, formant des fossés d'effondrement, dont le fossé de Sault-Aurel, mais aussi les fossés de Murs, de Lioux et de Banon. Dans les zones basses, fossés d'effondrement $\mathrm{NE} / \mathrm{SO}$ et synclinaux Est-Ouest (Jabron, Apt, Carpentras), s'installe un milieu lagunolacustre dont les dépôts détritiques et chimiques totalisent par endroits plusieurs centaines de mètres. Ainsi, dans le fossé de Sault vont se déposer en discordance sur les terrains crétacés, des argiles, des marnes et des calcaires en plaquettes.

9 Au début du Miocène, une nouvelle phase de compression ENE-OSO fait rejouer en décrochement certaines failles antérieures. En particulier, les failles qui délimitent le fossé de Sault vont avoir des déplacements de sens contraires. Ces mouvements antagonistes vont avoir pour effet de plisser les dépôts oligocènes du fossé selon une direction oblique par rapport aux accidents. Le fossé lui-même est rehaussé.

Enfin, la dernière, sinon la moindre, des phases tectoniques qui ont modelé la région intervient à la fin de l'Ere tertiaire, au Pliocène terminal. C'est de nouveau une compression nord-sud, déviation "provençale" de la phase paroxysmale de l'orogénèse alpine. La dalle calcaire, incapable de serrer beaucoup ses plis à grand rayon de courbure, va se laminer sur ses marges septentrionales et méridionales où elle est bien 
amincie et va chevaucher les synclinaux tertiaires correspondants. Ce mouvement s'accompagne d'un exhaussement général des reliefs et les fossés sont soulevés bien audessus du niveau des mers et lagunes pliocènes qui subsistent dans les paléo-vallées du Rhône et de la Durance.

11 Ce rapide rappel de l'histoire géologique de notre région nous permet d'analyser la morphologie actuelle, superficielle et souterraine. Nous voyons bien maintenant le contraste entre la zone déprimée du fossé de Sault, dont la position topographiquement basse a permis la conservation des sédiments tendres du Crétacé moyen et du Tertiaire, et les plateaux calcaires qui la délimitent, sur lesquels la roche mère subaffleurante ne supporte qu'un placage mince et discontinu de ses produits d'altération. La carte géologique de la figure 4 représente schématiquement les principaux terrains affleurants dans le fossé de Sault et ses abords immédiats.

Figure 4 : Carte géologique simplifiée du fossé de Sault et ses abords (d'après J.-M. Puig) Figure 4: Main springs of the Sault basin (after Derosier, 1981)

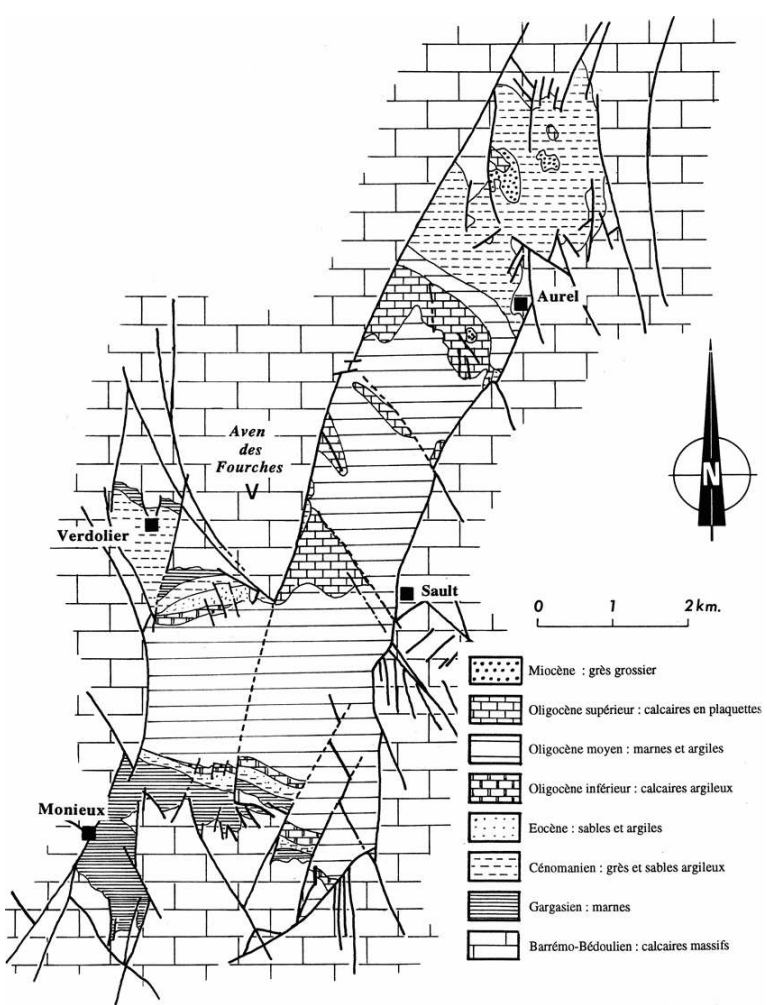

\section{Climatologie}

La région de Sault est soumise à un climat de type méditerranéen influencé par l'altitude, dont nous allons décrire ci-dessous les caractéristiques locales en utilisant les données de la station météorologique de Sault :

- la température moyenne interannuelle est un peu inférieure à 10 degrés $\left(9,92^{\circ}\right)$;

- l'influence méditerranéenne donne aux mois chauds des moyennes souvent supérieures à 20 degrés et des valeurs maximales atteignant 34 degrés ;

- l'influence montagnarde est responsable d'hivers rigoureux, avec des températures moyennes comprises entre 2 et 3 degrés pour les mois froids et plus de 110 jours de gelée par an en moyenne interannuelle ; 
- les deux influences interviennent sur le régime pluviométrique : la lame d'eau moyenne est de $960 \mathrm{~mm}$, la sécheresse estivale est bien moins marquée qu'en plaine, et si le début de l'automne est bien la saison la plus arrosée, la seconde période humide, intervenant selon les années entre février et juin, est donc "nivelée" par les moyennes mensuelles interannuelles.

\section{Ressources en eau}

13 Au plan hydrogéologique et hydrologique, le contraste est là encore bien marqué entre le fossé et les plateaux voisins : le fossé, dont le fond est resté tapissé par les marnes gargasiennes imperméables, dispose en toute saison de ressources en eau accessibles avec de nombreuses sources et le seul cours d'eau pérenne (la Nesque) à dix kilomètres alentour. Sur les calcaires, la fissuration provoque l'infiltration rapide des eaux météoriques, les vallons sont secs la plus grande partie de l'année, les sources sont rares, éloignées les unes des autres, et le plus souvent taries dès le début de la saison chaude, à l'exception notable de la source Millet située sur le versant sud de la montagne d'Albion.

Une quarantaine de sources a été recensée sur les quelques $20 \mathrm{~km} 2 \mathrm{du}$ fossé de Sault (Derosier 1981). La moitié coulait à la fin du mois d'août 1981, en étiage prononcé, et on peut donc les considérer comme pérennes. Bien sûr, beaucoup d'entre elles ne produisent en basses eaux que quelques litres à l'heure. Mais certaines, dont les sources de la Nesque, ont en toute saison un débit de plusieurs litres à la seconde. La carte de la figure 5 indique la situation et le débit des sources les plus importantes du fossé.

Figure 5 : Sources pérennes du fossé de Sault (d'après Derosier, 1981).

Figure 5: Main springs of the Sault basin (after Derosier, 1981).

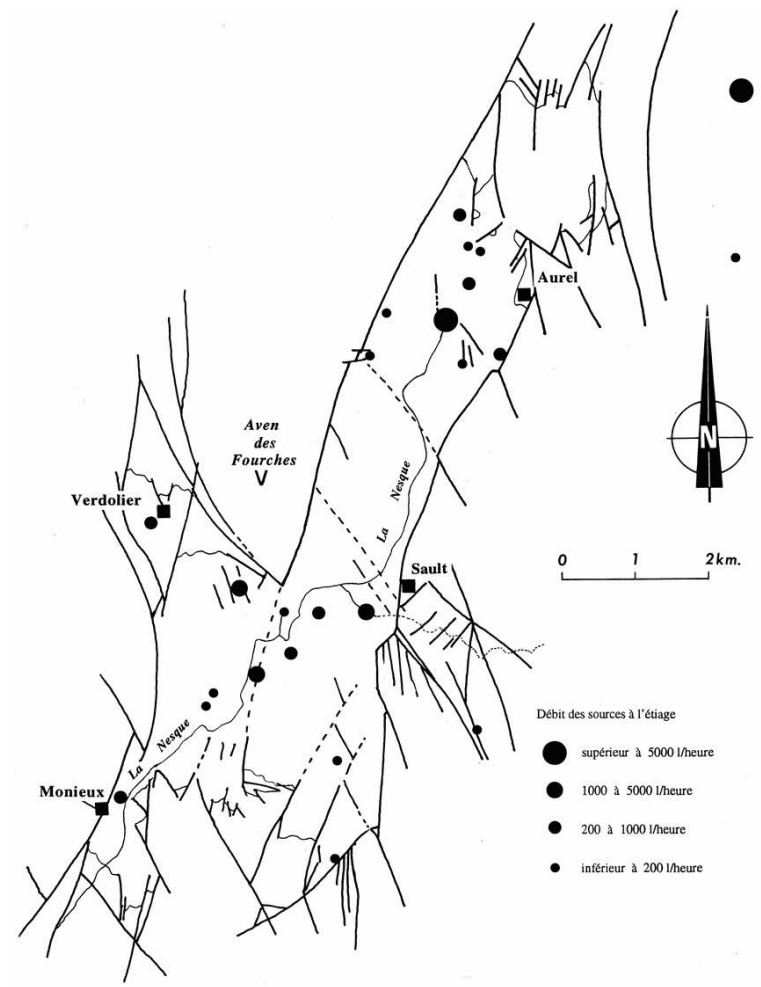




\section{Karstification}

Si la plaine du fossé, cultivable plus facilement et riche de nombreux points d'eau est plus attrayante, il faut se rapprocher des reliefs calcaires pour trouver des abris naturels, ceux que la karstification a creusé en agrandissant par dissolution les fractures de la roche. Même si l'érosion karstique a duré très longtemps et se poursuit de nos jours, elle ne donne pas partout les mêmes formes hypogées, et son action dépend d'un facteur énergétique : l'altitude par rapport au niveau de base, mer, lac ou fleuve récepteur final des eaux infiltrées. Sur les reliefs qui ont été rapidement exhaussés par rapport au niveau de base, le creusement a principalement une composante verticale. Se sont ainsi formés les grands avens des plateaux, qui se caractérisent par des successions de puits verticaux dont chacun peut atteindre ou dépasser 100 mètres de profondeur (167 m à Jean-Nouveau pour le puits d'accès). Les plus grandes cavités des plateaux de Vaucluse et d'Albion, dont quatre dépassent 500 mètres de profondeur et deux ont plus de 600 mètres de profondeur, montrent des galeries à grand développement. Mais ces galeries sont étagées entre 100 et 600 mètres de profondeur, après des puits d'accès très verticaux (Gaubert 1993). En revanche, les cavités situées à proximité immédiate du fossé de Sault ont vu se développer, sans doute lorsque ce fossé constituait le niveau de base local, des cavités de profondeur modeste et d'un développement horizontal relativement important.

\section{Le gisement}

\section{Description de l'endokarst et stratigraphie}

Compte tenu des difficultés d'accès aux parties profondes et en l'absence d'étude sédimentologique, les observations concernant l'endokarst et de son remplissage restent très sommaires. C'est donc sur le secteur de l'entrée et les parties accessibles du réseau que portera le premier niveau de description (fig. 6).

Figure 6 : Coupe schématique de l'aven des Fourches II et son remplissage karstique. Figure 6: Schematic section of the sinkholes of Les Fourches with karstic infilling.

OUEST

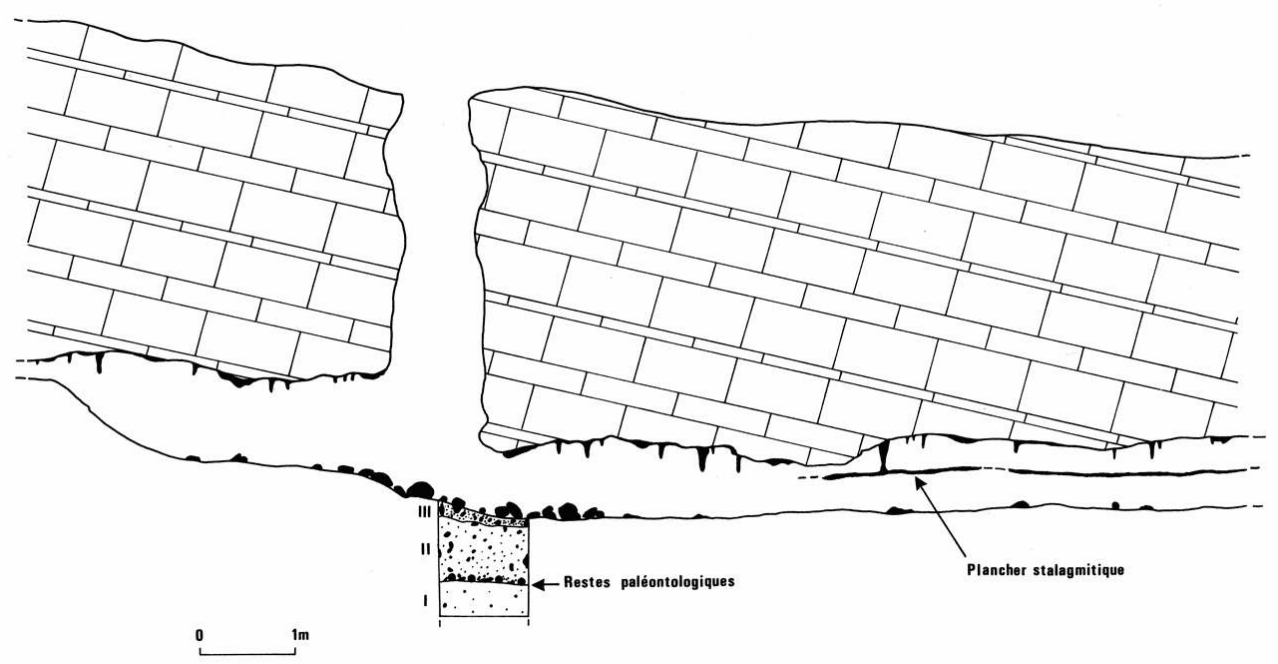


17 La seule entrée connue de la cavité se présente sous la forme d'un puits vertical de quatre mètres de profondeur, creusé dans les calcaires massifs barrémo-bédouliens, dont la largeur maximale n'excède jamais un mètre. Son diamètre à l'ouverture est de 80 centimètres. Ce puits permet d'accéder à une galerie d'orientation est-ouest, en grande partie horizontale, dont la hauteur dépasse rarement un mètre pour une largeur qui oscille, dans la partie explorée, entre 1,20 et $2 \mathrm{~m}$.

18 Concernant le conduit, quelques remarques essentielles peuvent être retenues: une dominante horizontale, une faible hauteur nécessitant la reptation, une obscurité totale, un taux d'humidité élevé avec d'importants ruissellements en période pluvieuse. Vers l'est, son développement atteint plusieurs dizaines de mètres (une trentaine a été reconnue). La partie ouest est quant à elle colmatée après quelques mètres.

19 Les parois ont gardé, dans l'ensemble, un important revêtement calcitique, même si par endroits, la déstabilisation de ce revêtement a entraîné la chute de nombreux fragments (phase de sédimentation primaire), conséquence d'un assèchement du climat et d'un abaissement des températures (faible dissolution et forte cryoturbation). Enfin, on observe la présence ponctuelle, vers l'est, d'un plancher stalagmitique suspendu de faible épaisseur.

Les observations stratigraphiques se limitent à des observations macrostratigraphiques effectuées à partir du remplissage mis en évidence dans un sondage de $1 \mathrm{~m} 2$ pratiqué à la verticale de l'ouverture. La base n'a pas été atteinte.

On observe de bas en haut:

- I- argile de décalcification rouge très plastique à cassure prismatique. Cette formation, reconnue sur une trentaine de centimètres d'épaisseur, est stérile. La fraction grossière, peu abondante, est essentiellement représentée par de petits galets karstiques et des rognons de silex d'origine locale affectés d'une patine noirâtre. De très rares fragments de revêtement calcitique, toujours de petites dimensions, sont visibles dans ce niveau.

- II- argile de décalcification rougeâtre emballant des fragments calcaires à des stades divers de dissolution (blocs décimétriques et cailloutis centimétrique, sables grossiers et sables fins), des spéléothems (fragments de stalactites, de stalagmites et de plancher stalagmitique) et des rognons de silex. C'est à la base de cet ensemble, épais d'une soixantaine de centimètres, et au contact du niveau sous-jacent, que les restes osseux et dentaires d'un équidé ont été mis en évidence.

- III- accumulation récente à la verticale de l'ouverture, constituée d'une matrice argileuse brune d'une dizaine de centimètres d'épaisseur, emballant des végétaux, de petits blocs calcaires anguleux et de nombreux restes de faune actuelle et subactuelle : Capra hircus, Ovis aries, Sus scrofa, Capreolus capreolus et lagomorphes dont Lepus europaeus. Ce type de vestiges est aussi abondamment représenté en surface.

\section{Observations sur la karstogenèse de l'aven de Fourches II}

L'aven des Fourches II, comme les avens environnants, donne accès à un conduit subhorizontal se développant à seulement quelques mètres sous la surface du plateau. La morphologie du plafond (présence locale de coupoles) indique un stade de creusement en régime noyé. Le remplissage, dont l'importance n'est pas connue, masque la morphologie initiale du conduit, ce qui ne permet pas d'établir s'il y a eu des phases de creusement en écoulement libre. La mise en place de ce type de conduit pourrait être contemporain de la phase de sédimentation du fossé de Sault (Oligocène l. s.), celui-ci 
formant alors un niveau de base d'altitude très supérieure à celle de Fontaine-deVaucluse, actuel exutoire du système aquifère (Puig 1987 ; Couturaud 1995).

Le plancher stalagmitique a été démantelé sous l'action de son propre poids et par le tassement des formations sous-jacentes, mais aussi par des écoulements qui ont érodé l'ensemble du remplissage. Un soutirage des sédiments vers des conduits inférieurs peut aussi être invoqué. Cette formation peut être rapprochée d'un autre plancher stalagmitique, localement suspendu, mis en évidence cette fois dans l'aven des Fourches I (Buisson-Catil et al. à paraître). La datation de ce spéléothème par la famille de l'uranium donne: 344,1 k.a. (+ inf/86,4)1. L'âge trouvé comprend l'infini dans sa limite d'erreur et est donc trop près de la limite de la méthode (350 000 ans) pour être pris tel quel en considération. Néanmoins, il faut noter que le rapport $234 \mathrm{U} / 238 \mathrm{U}$ plus grand que 1 signifie que l'équilibre n'est pas encore atteint et que l'échantillon est plus jeune qu'environ 1,5 million d'années. Le plancher stalagmitique des Fourches II, s'il est contemporain, comme on peut raisonnablement le supposer, de celui des Fourches I, se serait donc formé au plus tard durant le Troisième Interglaciaire avant le présent (Mindel-Riss de la chronologie alpine (350-300 Ka B.P. - stade isotopique 9).

Le plafond et les parois montrent des concrétions massives recristallisées, recouvertes très souvent par un concrétionnement plus récent avec des altérations de surface. Certaines concrétions sont cassées. La cassure présente une altération qui prouve son caractère ancien.

Les conduits accessibles par les avens des environs sont du même type. Deux phases de concrétionnement sont visibles. Des planchers stalagmitiques, parfois suspendus, indiquent un recreusement qui a évacué les remplissages détritiques. L'aven du Quartier du Ventoux $\mathrm{n}^{\circ} 2$ montre un soutirage au niveau d'un amoncellement de blocs, ce qui prouve que la karstification s'est développée plus en profondeur.

\section{Le cheval de l'aven des Fourches}

Quelques restes osseux et dentaires de grand mammifère proviennent des dépôts d'argile rouge plus ou moins riches en éléments détritiques (cf. supra). Ils concernent exclusivement les vestiges d'un herbivore, le Cheval (Equus caballus L., 1758), daté de la fin du Dernier Glaciaire (Tardiglaciaire). Deux espèces sont présentes au Pléistocène terminal : Equus caballus et Equus hydruntinus. Ce dernier, de plus petite taille, présentait des affinités avec les asiniens. On retrouve ces deux espèces de façon sporadique au début de l'Holocène (Postglaciaire). Les dimensions et la morphologie de nos pièces permettent rapidement d'écarter le cheval hydruntin et d'attribuer ce matériel au cheval caballin.

\section{Matériel et datation}

Le matériel est réduit en raison de la faible surface explorée; un total de 15 éléments $(\mathrm{NRD}=30)$, plus quelques esquilles, se rapportent à un individu unique de cheval juvénile. Les pièces les plus caractéristiques sont :

- deux séries dentaires (dents isolées) supérieures : la série droite comprend la P2 et la M1, et la série gauche plus complète de la P2 à la M3 (cette dernière cassée avec partie vestibulolinguale manquante) avec deux dents lactéales coiffant la P3 et la P4 (fig. 7) ; 
- dents isolées : I2 supérieure droite, M1 inférieure droite, P2 inférieure gauche, deux incisives lactéales, deux fragments d'une dent inférieure ;

- le post-crânien est représenté par des fragments de côtes, un radius droit incomplet (l'extrémité distale et la moitié latérale de l'extrémité proximale manquent), un métacarpien III droit complet, une phalange I complète (extrémité proximale abîmée), un trapézoïde droit, un fragment proximal de métacarpien, un fragment de condyle distal de fémur, un tibia droit (manque l'extrémité proximale).

Figure 7 : Aven des Fourches II.

Figure 7: Les Fourches II.

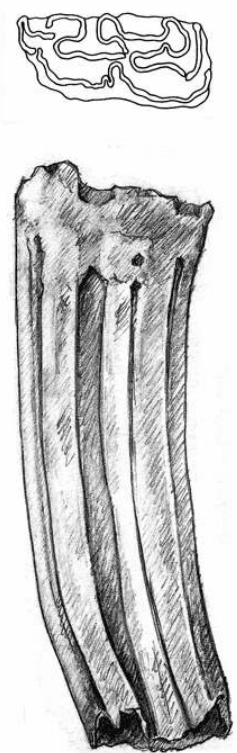

1
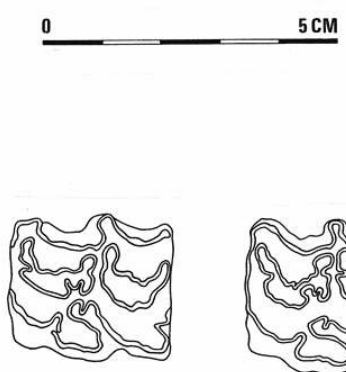

2
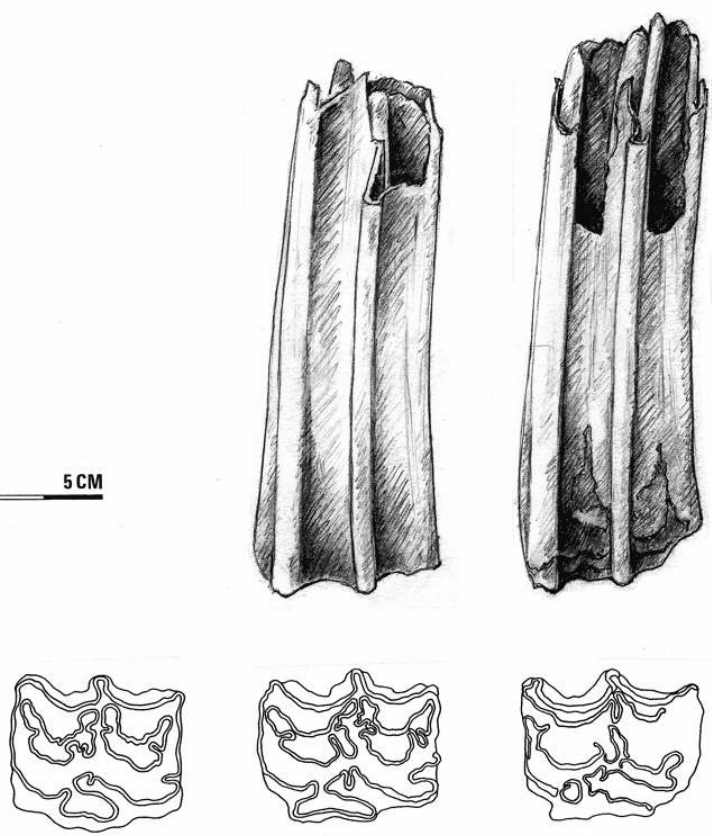

3

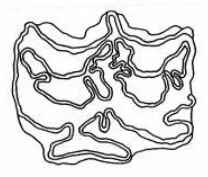

4

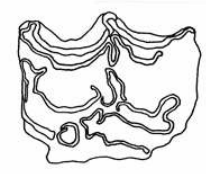

5

$1: M 1$ inférieure droite (dent isolée). 2 à 5, série dentaire supérieure gauche. $2: \mathrm{dp} 3 ; 3: \mathrm{dp} 4 ; 4: \mathrm{M} 1$; $5: \mathrm{M} 2$.

7-lower right molar (isolated tooth), 2 to 5 - upper left dental serie, $2=d P 3 ; 3=d P 4 ; 4=M 7 ; 5=M 2$.

(Dessin F. Chardon)

(drawings F. Chardon)

Les dimensions (en millimètres) des dents et des ossements sont données dans les tableaux 1 et 2 . 
Tableau 1 : Equus caballus de l'Aven des Fourches II (mesures en mm). Table 1 : Equus caballus from Les Fourches II (measurements in $\mathrm{mm}$ ).

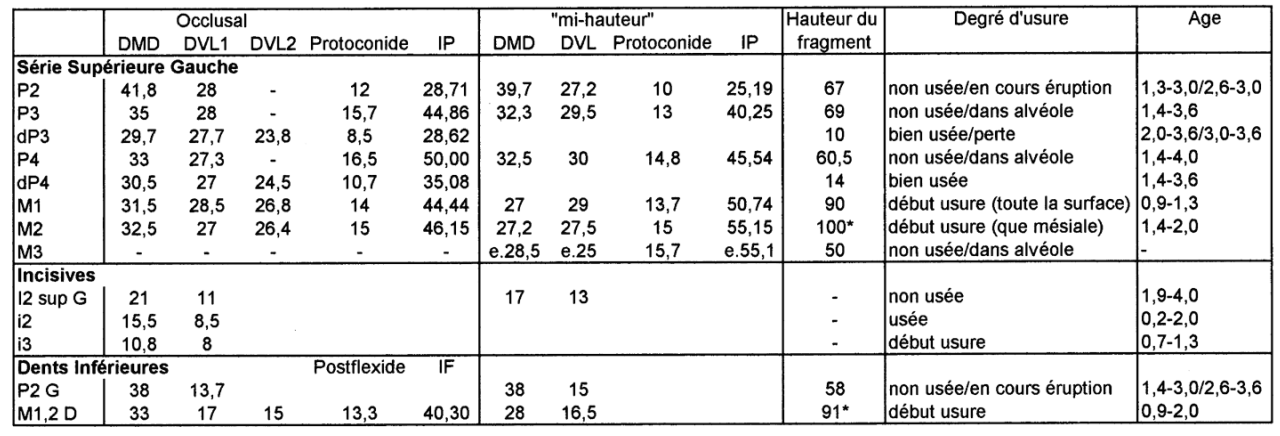

$\mathrm{DMD}=$ diamètre mésio-distal $; \mathrm{DVL}=$ diamètre vestibulo-lingual $(1:$ avec cément $; 2:$ sans cément $)$ $\mathrm{IP}=$ indice protoconide ; IF = indice postflexidique ; ${ }^{*}$ hauteur totale de la couronne. Age d'après $\mathrm{M}$. Levine, 1982 (ans,mois : ex. 2,6 =deux ans et six mois).

$D M D=$ mesio-distal diameter; $D V L=$ vestibulo-lingual diameter (1: with cement; 2 : without cement); IP= protoconid index; IF= postflexidic index; * total crown heigh. Age according to Levine, 1982 (years months: ex. 2.6=two years and six months).

Tableau 2 : Equus caballus de l'Aven des Fourches II (mesures en mm). Table 2 : Equus caballus from les Fourches II (measurements in $\mathrm{mm}$ ).

\begin{tabular}{|c|c|c|c|}
\hline & Métacarpien D & & Phalange I ant. \\
\hline L. totale & 237 & \multirow{11}{*}{$\begin{array}{l}\text { H.totale } \\
\text { H. ant. } \\
\text { H. post. } \\
\text { D.T. prox. } \\
\text { D.A.P. prox. } \\
\text { D.T. milieu dia. } \\
\text { D.A.P. milieu dia. } \\
\text { D.T. susart.dist. } \\
\text { D.T. art.dist. }\end{array}$} & 83,5 \\
\hline L. externe & 229 & & 78 \\
\hline D.T. max. prox. & 53 & & 77,5 \\
\hline D.T. art. prox. & 52 & & e.53,5 \\
\hline D.A.P. prox. & 35 & & 35,7 \\
\hline D. fac. magnum & 43 & & 34,5 \\
\hline D. fac.ant.unciforme & 13,5 & & 25 \\
\hline D.T. milieu dia. & 34,5 & & 45,5 \\
\hline D.A.P. milieu dia. & 27 & & e.42 \\
\hline D.T.susart.dist. & 47 & & \\
\hline D.T. art.dist. & e.44,5 & & \\
\hline D.A.P. susart.dist. & 28 & & Tibia D \\
\hline D.A.P. art.dist. & 34,5 & \multirow{5}{*}{$\begin{array}{l}\text { D.T. mini.dia. } \\
\text { D.A.P. mini.dia. } \\
\text { D.T. dist. } \\
\text { D.A.P. dist. }\end{array}$} & 42,5 \\
\hline \multirow[t]{3}{*}{ D.A.P. max.cond.int. } & 28,7 & & 33,5 \\
\hline & & & $>=74$ \\
\hline & Trapezoide D & & 47 \\
\hline \multirow{4}{*}{$\begin{array}{l}\text { H. max. } \\
\text { D.T. max. } \\
\text { D.A.P. max. }\end{array}$} & 23,5 & & \\
\hline & 20,7 & & Calcaneum D \\
\hline & 32,3 & \multirow{3}{*}{$\begin{array}{l}\text { D.T. mini.corps } \\
\text { D.A.P. mini.corps } \\
\text { D.T. max. } \\
\text { D.A.P. niveau bec }\end{array}$} & $\begin{array}{c}19 \\
\text { e. } 40,5\end{array}$ \\
\hline & Radius D & & 55 \\
\hline $\begin{array}{l}\text { D.A.P. max.prox. } \\
\text { D.T. milieu dia. } \\
\text { D.A.P. milieu dia. }\end{array}$ & $\begin{array}{c}e .47,5 \\
39,5 \\
30\end{array}$ & & 52 \\
\hline
\end{tabular}

$\mathrm{L}=$ =longueur ; D.T. =diamètre transversal ; D.A.P. =diamètre antéro-postérieur ; fac. =facette ; H. =hauteur ; art. =articulaire; susart. =susarticulaire; prox. = proximal; dist. =distal ; dia. =diaphysis ; ant. =anterieur ; D. =droit.

L.=lenght; $D . T .=$ transversal diameter; D.A.P.=antero-posterior diameter: fac. $=$ facet; $H_{\text {. }}=$ heigh; art. =articular; susart. $=$ susarticular; prox. $=$ proximal; dist. $=$ distal; dia. $=$ diaphysis; ant. =anterior; $\mathrm{D} .=$ right.

Une quinzaine d'esquilles complète cette liste. Un certain nombre d'entre elles (d'un poids de $104 \mathrm{~g}$ ) a fait l'objet d'un datage $14 \mathrm{C}$, effectué au Laboratoire de Géologie du Quaternaire alors à Marseille-Luminy (mars 1994). Les esquilles osseuses fournissent 
suffisamment de matière organique (collagène) pour donner un résultat satisfaisant. Un âge de $12500 \pm 300$ ans B.P. (LGQ-1069) indique la fin du Pléistocène et du Dernier Glaciaire (Würmien). Cette période tardiglaciaire montre une succession de phases climatiques plus ou moins contrastées conduisant à l'Interglaciaire actuel (Holocène). L'intervalle de temps 12 800-12 200 se rapporte à la fin d'une phase d'amélioration climatique, humide et fraîche, le Bölling (13 300-12 300), précédant une courte phase froide (Dryas II, 12 300-11 800) et suivie à nouveau d'un réchauffement (Alleröd, 11 800-10 800). Cette dernière oscillation est parfois considérée comme marquant la fin du Paléolithique avec la transition vers d'autres industries épipaléolithiquesmésolithiques. De même, il est maintenant courant de considérer la période 13 300-10 800 comme une même phase climatique appelée Interstade Bölling-Alleröd (Magny 1995).

Durant le Dernier Glaciaire, plusieurs sous-espèces de Cheval se succèdent : germanicus au Würm ancien, puis gallicus et arcelini au Würm récent. La sous-espèce arcelini a récemment été décrite (Guadelli 1991) à partir du matériel des niveaux magdaléniens de Solutré (Saône-et-Loire), niveaux datés de $12580 \pm 250$ ans B.P. (LY-393). Ce travail servira largement dans les comparaisons qui suivent afin de préciser l'attribution taxinomique du cheval de Sault.

\section{Eléments de taphonomie}

Les dents sont relativement bien conservées, trouvées en connexion lâche, sans os mandibulaire ou maxillaire, alors que les ossements de couleur claire, blancs à grisâtres, présentent une surface osseuse légèrement desquamée (cupules de dissolution), montrant de fines fissurations et un début de "digestion" des masses spongieuses des épiphyses. Des traces d'impact naturel sous forme de dépressions mal délimitées sont observables et des marques de dents de rongeurs sont visibles le long du bord médial de la diaphyse du radius; ces marques indiqueraient un rongeur de taille moyenne (comme par exemple le rat-taupier Arvicola). Le bord des fractures, comme les surfaces osseuses, est émoussé. Ces caractéristiques taphonomiques indiquent une relative dispersion, accompagnée de fragmentation, des éléments osseux qui se sont trouvés exposés assez longtemps à l'air libre, avec l'intervention d'un rongeur, avant l'enfouissement dans les dépôts colluviés. Le contexte topographique (aven, dimension de l'ouverture) et la situation des ossements (aplomb de l'ouverture), le type d'éléments trouvés et l'état du matériel (os complets, degré d'altération), permettent d'avancer que le Cheval de Sault concerne un individu piégé naturellement dans le karst. Des accumulations de faune dans les aven-pièges sont bien connus (ex. : aven des Planes à Monieux, Aujard-Catot et al. 1979), et concernent souvent de larges populations d'herbivores grégaires. Il est étonnant ici qu'un seul sujet soit présent, ce qui pourrait simplement s'expliquer par les dimensions modestes du sondage effectué.

31 Un premier examen de ce matériel montre rapidement que nous avons affaire à un poulain dont nous avons essayé d'estimer l'âge. Ce facteur est important dans la détermination de matériel paléontologique, détermination nécessitant la prise de mesures en vue de comparaisons biométriques avec d'autres populations ou spécimens. Les estimations de l'âge des mammifères sont fondées sur le degré d'éruption et d'usure dentaire et sur le degré d'épiphysation des os longs des membres (auxquelles il faudrait ajouter la morphologie des bois chez les cervidés). 

celle observée chez d'autres grands herbivores (seul le tempo diffère suivant la taille des espèces considérées). Chez le Cheval, les premières molaires (M1 et M2) sortent les deux premières années suivies par la deuxième prémolaire vers la fin de la troisième année (avec perte de la première lactéale $\mathrm{dP} 2$ ) ; les autres prémolaires ( $\mathrm{P} 3$ et $\mathrm{P} 4$ ) sortent entre 3 et 4 ans (perte respective de $\mathrm{dP} 3$ et $\mathrm{dP} 4$ ) et la série se met définitivement en place avec l'éruption de la troisième molaire jusque vers 5 ans. Ainsi, à 4-5 ans le sujet possède sa dentition définitive; un poulain désigne un individu de 0-3 ans, qui deviendra ensuite un jeune adulte capable de se reproduire. Les mâles se groupent alors en bandes (célibataires) éloignées des harems dominés par un étalon plus vieux et plus fort. d'usure dentaire à partir d'espèces ou races domestiques (Lévine 1982). Le degré d'usure de chaque dent de la série dentaire supérieure, et des dents isolées, du cheval de Sault est indiqué dans le tableau 1 avec la fourchette d'âge retenue. La série dentaire supérieure présente des M1 et M2 en début d'usure, le reste des dents définitives étant non usées et les P3 et P4 sont encore coiffées des dents lactéales bien usées (fig. 8). Les dents isolées (inférieures et incisives) montrent également une usure nulle ou faible, ce qui démontre l'appartenance du matériel à un même individu. La conjonction de l'ensemble des intervalles de temps d'éruption et d'usure permet de retenir un âge élargi, entre 0,9 et 2 ans et, plus précisément, autour de 1,3-1,6 $\mathrm{an}^{2}$. L'absence de racines nettement constituées (M1 et M2) et la fragilité de la couronne non encore formée dans la partie basale (PM, M3) contribuent à cette estimation ; le développement des racines commençant à partir de 2 ans jusqu'à presque 8 ans.

Figure 8 : Sault-Aven des Fourches II. Série dentaire supérieure gauche de la P3 à la M3 avec deux dents lactéales coiffant la P3 et la P4.

Figure 8 : Sault-Aven des Fourches II. Upper row tooth, with P3 to M3 and two lacteal teeth on P3 and P4

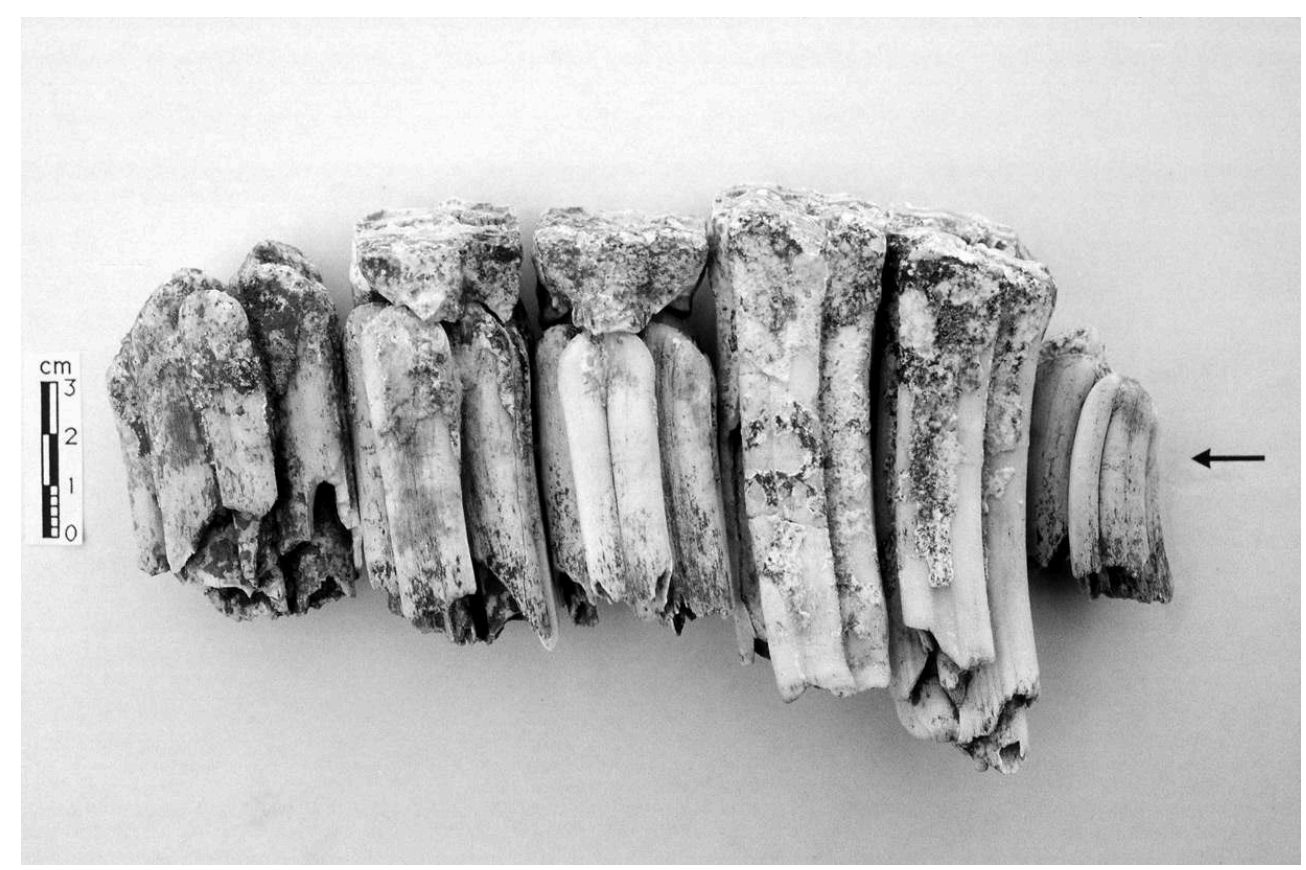

(Cliché J. Buisson-Catil)

PALEO, 13 | 2001 
34 les chevaux sont pour les os concernés : radius proximal $=$ entre 1,3 et 1,6 an; métacarpien III distal $=$ autour de 1,3 an ; phalange I proximal $=1-1,3$ an ; tibia distal $=$ autour de 2 ans; calcaneum = autour de 3 ans. Tous les os de l'aven des Fourches II montrent un début d'épiphysation avec des traces de suture visible entre diaphyse et épiphyse, traces en particulier assez nettes sur le tibia. La partie proximale du calcaneum (tuber) est manquante et il est intéressant de relever que des phénomènes de digestion osseuse sur l'ensemble des os longs se placent préférentiellement sur les sutures épiphysaires. La taphonomie (degré d'altération) vient ainsi compléter ces observations qui confirment l'âge du cheval de Sault aux alentours de 1,3-2 ans.

L'ensemble de ces données permet de connaître la saison à laquelle le cheval de Sault a été piégé dans l'aven. Chez les grands herbivores, la période de naissance se situe au printemps (avril-mai). Considérant l'âge estimé ci-dessus, le poulain est tombé dans l'aven pendant l'automne ou au début de l'hiver.

\section{Description et comparaison}

La présence d'un sujet juvénile constitue un certain handicap dans la caractérisation morphométrique; de plus, nous n'avons ici qu'un individu et non une série (i.e. population). Il a souvent été signalé le danger de prises de mesures et leur utilisation sur les dents isolées non ou peu usées, ce qui a amené des auteurs à les mesurer selon un point déterminé (pour le Cheval il s'agit du point $\mathrm{P}$ à $2 \mathrm{~cm}$ de la divergence des racines, ce qui n'est guère possible ici). Seule la série supérieure gauche, la plus complète, a été mesurée au niveau occlusal et à mi-hauteur de la couronne sur la série placée en connexion (c'est-à-dire sur le même plan horizontal : soit environ $3 \mathrm{~cm}$ audessous de la surface occlusale sur les PM et la M3 et environ 4-4,5 cm sur les M1 et M2) $(\leftarrow$ fig. 8).

37 Les dents supérieures présentent une morphologie typiquement caballine avec des styles marqués (surtout para- et méso-styles), arrondis sur les molaires et dédoublés sur les prémolaires; ce dédoublement est particulièrement fort sur le parastyle des $\mathrm{P} 3$ et $\mathrm{P} 4$ et sur le mésostyle des $\mathrm{P} 2$ et $\mathrm{P} 3$. Les faces interstylaires sont nettement concaves et le protocône est long avec un sillon lingual très marqué, allant en s'évasant dans le bas des couronnes. L'hypsodontie est forte (cf. hauteur de la M2 supérieure et M1, 2 inférieures); le pli caballin est simple et bien marqué sur la M1 supérieure et les fossettes montrent des plissotements importants et relativement fins (on rappellera de nouveau qu'il s'agit d'une dent en début d'usure). Les deux dents lactéales possèdent aussi des styles développés, un pli caballin net et des plis sur la préfossette.

Les dimensions prises à mi-hauteur des dents de la série supérieure (tabl. 1) sont comparées aux mesures prises au niveau occlusal (données in Guadelli 1991). Les comparaisons concernent les sous-espèces germanicus (Combe-Grenal, Pair-non-Pair), gallicus (Camiac, Jaurens, Solutré, Saint-Germain-la-Rivière) et arcelini (Solutré). Les dents jugales du cheval de Sault sont particulièrement fortes et se rapprochent des moyennes obtenues sur E. c. germanicus; les mesures dépassent souvent la variation métrique observée sur les populations de chevaux du Würm récent. L'indice protoconique (IP) augmente de $\mathrm{P} 3$ à M2 et la M3 à un IP proche de M2 : cette disposition serait plus fréquente chez les chevaux d'âge ancien, alors que l'IP moyen des M3 est toujours plus faible que celui des molaires chez les formes plus récentes (Guadelli 1991). 
Les dents isolées inférieures indiquent également un appareil masticatoire puissant pour le cheval de Sault.

Les éléments post-crâniens permettent de préciser ces dernières observations. Le métacarpien a une longueur totale de $237 \mathrm{~mm}$. Les valeurs (en $\mathrm{mm}$ ) pour les chevaux sont les suivantes (Guadelli 1991 : tabl. 30-32) :

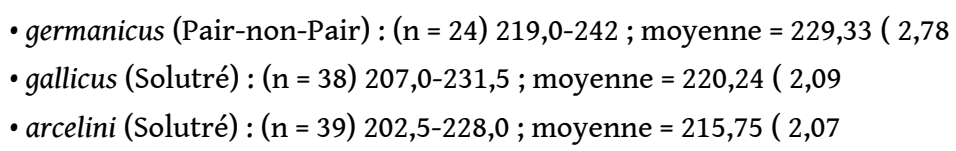

La longueur de notre métacarpien sort complètement des intervalles de variation des chevaux récents, alors qu'elle est proche des valeurs de E. c. germanicus. Les autres mesures, à l'exception peut être du DT proximal et du DAP sus-articulaire distal, sont plus faibles et rejoignent les valeurs du groupe gallicus-arcelini. De même, les autres ossements ont souvent des valeurs proches de celles de ce groupe, mais il faut considérer que les os longs ont souvent des mesures généralement faibles quelle que soit la sous-espèce considérée. Les hauteurs de la phalange I sont plus proches des valeurs de germanicus. La faiblesse des mesures transverses à l'axe des os dénote essentiellement la "jeunesse" de notre individu.

Le Poulain fossile de l'aven des Fourches II était un animal robuste avec des dents jugales puissantes et une hauteur au garrot estimée, à partir du métacarpien (coefficient de Kiesewalter), à $1,52 \mathrm{~m}$, probablement proche de sa taille adulte. Pour comparaison, on citera la taille moyenne chez gallicus égale à $1,41 \mathrm{~m}(1,24-1,60)$ et chez arcelini égale à $1,38 \mathrm{~m}(1,21-1,57)$ (Guadelli 1991). Ces caractéristiques morphométriques permettent de le rapprocher de formes anciennes telles que Equus caballus germanicus du Würmien ancien. Avec réserve, au regard de l'âge récent de ces restes, nous désignerons le cheval de Sault sous le nom Equus caballus aff. germanicus.

\section{Les derniers chevaux sauvages en Provence}

Dans un essai de synthèse et afin de saisir la dynamique du peuplement des chevaux dans le Sud-Est de la France, nous avons cherché les gisements, provençaux et régions périphériques (Languedoc, Dauphiné-Alpes, Italie), d'âges tardiglaciaire et postglaciaire ayant livré des restes de Cheval caballin.

La fin du Dernier Glaciaire (Würmien) est marquée par la régression des glaciers alpins qui s'amorce vers 18-16 000 ans et entraîne le désenglacement des grandes vallées de nos régions (Jorda 1988). Le Dryas ancien (Dryas I, entre 15000 et 13 000) est encore froid et voit le développement d'une steppe à armoises et fruticée, à genévriers. La phase de réchauffement qui suit, regroupant le Bölling jusqu'à l'Alleröd, est considérée comme une transition avec une reconquête forestière des zones de moyenne altitude (pineraies). Ces périodes tempérées se manifestent souvent par une phase d'érosion et l'absence, ou la rareté, de dépôts en Provence : par exemple à l'Adaouste (Bouches-duRhône) et au Rainaude 1 dans le Var (Onoratini 1982). Une nouvelle occurence froide assez brève est de nouveau observée au Dryas récent (entre 10800 et 10000 env.) suivie par l'installation définitive de la végétation postglaciaire mésophile.

Cette période voit le développement de cultures et faciès originaux, à partir ou indépendamment d'un substrat magdalénien: Salpétrien en Languedoc, ArénienBouvérien et Gravettien-Epigravettien en Provence et en Italie; le Magdalénien 
supérieur ou terminal est également présent. L'Azilien, le Montadien, le Valorguien (Romanellien provençal), le Sauveterrien ou le Castelnovien caractérisent des industries, plus ou moins de transition, de l'Epipaléolithique-Mésolithique dans le SudEst de la France qui précèdent le Néolithique, du Cardial au Campaniforme (BuissonCatil, Sauzade 1995).

L'existence du Cheval caballin est relevée dans plusieurs gisements du Pléistocène terminal et du Postglaciaire dans le sud-est de la France. Les restes ne sont cependant jamais trouvés en grande quantité (souvent de l'ordre d'une dizaine, ou moins !) ce qui a amené nombre d'auteurs à juste les signaler, limitant d'autant les comparaisons taxinomiques. Les dates $14 \mathrm{C}$ qui suivent sont exprimés en "années B.P." et sont publiées pro parte dans M. Schvoerer et al. (1977), accompagnées du nombre de restes (n) découverts.

Le Cheval semble régulièrement présent dans les associations animales autour du maximum glaciaire, associé au Renne et/ou au Bouquetin : c'est le cas à la Salpétrière couche $6 \mathrm{~b}(\mathrm{n}=45 ; 19165 \pm 200), \mathrm{d}(\mathrm{n}=42 ; 18970 \pm 280)$ et $5(\mathrm{n}=8 ; 17960 \pm 600)$ - et à la Baume d'Oullins (c. 9) dans le Gard et à l'aven Bouet dans l'Hérault (15 $460 \pm 190)$ (Brugal 1981, 1982). En Provence, il est présent dans le Vaucluse à l'aven des Planes (plusieurs individus: Aujard-Catot et al. 1979) et dans le Var à la grotte du VieuxMounoï (deux individus entiers, $18400 \pm 1700$; Helmer inédit), à la Bouverie (c. 3 autour de 23000 ans, $\mathrm{n}=2$ dents) et au Rainaude $1($ c. $5, \mathrm{n}=64$ ) (Bonifay in Onoratini 1982). Les sujets du Mounoï correspondent à un individu adulte (Ht. au garrot de 1,44 m) et un jeune (Ht. au garrot de 1,42 m). Dans les premiers sites, le Cheval est proche de la sousespèce gallicus alors qu'il apparaît plus archaïque et plus robuste dans les gisements varois de la Bouverie et de Rainaude (E. cf. germanicus).

Durant le Bölling-Alleröd, le Cheval devient rare et on le signale en Provence à l'Adaouste ( 5 restes dans la couche 17 datée de $12760 \pm 250$ et $12981 \pm 266 ; 1$ reste dans la couche 12 datée de $12280 \pm 190$ et $12054 \pm 370$ ), dans le Vaucluse à Chinchon 1 (c. 15/ A, $12000 \pm 420$ ) et Chinchon 5 (c. 12-15) (Brochier 1977), dans le Var au Rainaude 1 (c. 3 $\mathrm{n}=3$ et c. $1, \mathrm{n}=3)$. Il est également présent en Languedoc à la Salpétrière (c. $3, \mathrm{n}=2$, $13100 \pm 100)$ et dans le Dauphiné à l'abri du Calvaire, Isère (13 $450 \pm 300,12970 \pm 300)$ (Desbrosse 1976) et dans la grotte du Tai et l'abri du Campalou dans la Drôme (couche inférieure surmontée d'une couche datée de $12800 \pm$ 300) (Brochier in Desbrosse 1976).

L'extrême fin du Tardiglaciaire et le tout début du Postglaciaire (entre 11000 et 8000 ans) voit l'installation de cultures transitionnelles épipaléolithiques-mésolithiques ou plus nettement mésolithiques. Le gisement de Cornille (Bouches-du-Rhône) montre une séquence intéressante ayant fourni des restes bien étudiés de chevaux (Bonifay 1968 ; Bonifay, Lecourtois-Ducgoninaz 1976). La couche 10 (10 $540 \pm 310$ et $10870 \pm 320)$ livre 8 restes, la couche $9(10270 \pm 470) 3$ restes (c. 10-9 : Romanelien ; la couche 6 (8 $100 \pm 130$ et $7000 \pm 130$ ) fournit 15 restes et la couche 4 , la plus riche, contient plus de 50 restes (c. 6-4: Montadien). Les conclusions décrivent les chevaux de Cornille comme étant de petite taille (dents de petites dimensions, en particulier pour les prémolaires), se rapprochant de la limite inférieure de E. caballus gallicus.

Des éléments d'une espèce proche sont également présents dans l'Epipaléolithique du Vaucluse : à Unang $(\mathrm{n}=2)$, Gramari (c. 5-7 datées de $10120 \pm 230, \mathrm{n}=9)$, à Soubeyras et à Roquefure $(n=1)$ (Paccard et Bouville 1993; Poulain 1971; Brochier 1977; Helmer, inédit) ; et, dans le Var, à la Baume Goulon (Bonifay 1978). Il est également cité dans le Gard à la Baume de Valorgues (c. 8, n = 4, datée de $10970 \pm$ 85) (Bonifay 1978). 
50 Rhône à Fos-sur-Mer, site montadien du Mourre-Poussiou ( $n=18,8980 \pm 200$; Lecourtois-Duc Goninaz 1984); et, en Vaucluse, dans le Sauveterrien de Gramari (Poulain 1971): couche 3c $(n=14)$, couche $3 b(n=8)$ et couche $3 a(n=1$, datée de $8000 \pm$ 190) (la couche 4 sous-jacente est datée de $9340 \pm 220$ ). En Languedoc, le site gardois du Plaisir, daté d'environ 8500 ans, livre quelques rares éléments (Brugal in Bazile et al. 1986-87). Le Cheval disparaît par la suite du sud de la France et ne fera sa réapparition dans nos régions que 2 à 3 millénaires plus tard. Frigouras à Peipin dans les Alpes de Haute-Provence : structure $35(n=1,5450 \pm 100)$ (Helmer in Buisson-Catil et al. 1991). Il est aussi rare dans les gisements chasséens de l'Ardèche comme Combe Obscure (c. 5, $\mathrm{n}=2$ ) et vauclusiens (Unang; $\mathrm{n}=1$ ), dans la grotte de l'Eglise supérieure dans le Var $(\mathrm{n}=4)$ et sur le site des Calades à Orgon dans les Bouches-du-Rhône (ce dernier campaniforme, $n=4$ ) (Helmer 1991). Il ne devient réellement abondant que dans le gisement néolithique final (Campaniforme) de La Balance à Avignon (Helmer 1979 ; env. 4 500-3 800). Dans ces sites, il s'agit d'une espèce de petite taille, voisine du Cheval de Solutré (Helmer 1979, 1991), égale ou légèrement plus grande que celle du Cheval du Néolithique final de Roucadour (Lot). Sur les Causses, une population (27 individus) a été décrite par P. Ducos (1957) représentant un Cheval de moyenne taille proche du type des forêts et qui pourrait désigner des animaux déjà domestiqués. P. Ducos (1960) note également la présence du Cheval dans les gisements néolithiques (essentiellement chasséens, mais contextes mélangés, Courtin 1974) des grottes Barriéra (Alpes-Maritimes), Saint-Benoit (Alpes de HauteProvence) et dans le Lot à Reilhac et à l'abri Pagès. Il est signalé dans les sites Fontbouisse de l'Hérault (Le Lébous, Gravas), le Campaniforme tardif de St-Côme-deMaruéjols, Gard (Courtin 1978) et le Plan-des-Vaches, Bouches-du-Rhône ( $\mathrm{n}=3$, Helmer 1982). Quoiqu'il en soit sur le statut de ces dernières formes (voir Courtin 1978), les chevaux sauvages semblent absents à partir de l'Age du Bronze ${ }^{3}$ alors que les premières formes domestiques apparaissent. Rappelons ici que la plus ancienne preuve de domestication du Cheval se trouve en Europe de l'Est - site de Dereivka en Ukraine, autour de $6000-5400$ ans - (Helmer 1992). Globalement, le Cheval reste un élément rare des faunes sauvages du Néolithique et du Chalcolithique en France, toujours trouvé en petite quantité dans une cinquantaine de gisements (liste in Poplin et al. 1986). Dans le Sud-Est son utilisation domestique devient effective au Bronze final et surtout à l'Age du Fer (Courtin 1978).

L'Italie présente une situation globalement proche du Sud-Est de la France et le Cheval parait absent ou peu abondant. Il existe très peu de gisements contenant du Cheval à la fin du Pléistocène supérieur et à l'Holocène ancien (Gravettien-Epigravettien) en Italie nord-orientale (environ 5 cités in Bon et al. 1991) et à peine plus en Italie centrale (environ une dizaine in Caloi, Palombo 1994a et b). Dans une synthèse récente sur la transition Pléistocène-Holocène, les auteurs concluent: "During the time span correlated with the Dryas I, the equids are not reported from Northern Italy and seem to have been quite rare elsewhere. They kept rare throughout the rest of the Lateglacial and apparently died out in the course of the Dryas III interval" (Masseti et al. 1996, p. 91). Il n'y a pas de précision sur le statut taxonomique des formes italiennes. 


\section{Discussion}

53 quelques considérations générales peuvent être présentées, d'une part sur l'occurence du cheval de Sault, d'autre part sur l'histoire plus générale des chevaux dans le sud-est de la France.

L'aven des Fourches II, localisé à près de $800 \mathrm{~m}$ d'altitude, livre des restes d'un cheval dont les caractéristiques morphométriques le rapprochent des formes du Würmien ancien, E. c. germanicus. Une datation sur ossements place cet individu dans l'interstade tempéré-humide de Bölling-Alleröd, soit le Würm récent. Seuls quelques rares éléments d'âge plus ancien seraient comparables (Bouverie, Rainaude) alors que tous les gisements, quand l'effectif le permet, démontrent la présence d'un cheval de plus petite taille, proche des chevaux de Solutré; ceci depuis le Pléistocène terminal jusqu'au Postglaciaire.

Trois raisons peuvent expliquer cette découverte étonnante :

- Problème de datation. Un âge Pléistocène est en accord avec la stratigraphie (cf. supra) : les pièces se trouvent en place dans des sédiments anciens (argile rouge) et leur fossilisation est fort différente des restes récents relevés en subsurface. L'âge fourni par le $14 \mathrm{C}$ a pu être rajeuni sous l'effet de ruissellement et de dégradation de matière organique ; cependant le résultat obtenu, avec une fourchette d'erreur faible, a été jugé satisfaisant (R. Lafont, comm. pers.).

- Les équidés sont reconnus pour présenter une grande souplesse adaptative en relation avec l'alimentation et les substrats (sols). Ces caractères concernent l'importance des plissements d'émail dentaire et les dimensions des os distaux des membres (métapodes, phalanges) qui s'élargissent plus ou moins (DT, DAP). La taille elle-même est sujette à variations en fonction de paramètres climatiques. Toutefois, le cheval de Sault présente de faibles plis d'émail et une certaine gracilité, malgré son âge ontogénique, qui ne permet pas d'affirmer une telle cause. Il faut également noter que le Cheval de l'aven des Planes près de Monieux (alt. 750 $\mathrm{m}$ ), attribué au maximum glaciaire et partageant avec Sault le même environnement de plateaux calcaires, désigne un cheval de taille proche de celui de Solutré. Il est vrai que le polymorphisme écologique des formes équines est réel, et pourrait être retenu pour expliquer la présence d'un Cheval de grande taille à Sault, d'autant plus que la fin du Pléistocène subit des changements climatiques significatifs et voit le morcellement géographique de nombreuses formes qui tendent à disparaître (soit par extinction, soit par changement de leur aire de distribution). Ce dernier point nous entraîne vers une troisième hypothèse.

- Il existe un conservatisme des formes anciennes en Provence. Cet "endémisme" s'expliquerait par la géomorphologie de cette province, bordée par des barrières naturelles qui retreignent les échanges (mer au sud, Rhône à l'ouest, Alpes - plus ou moins englacées au nord et à l'est). Si l'arrivée de chevaux de petite taille (type Solutré) se place en Provence dès le début du Würm récent, elle aurait pu repousser ou isoler des populations plus anciennes, continuant à exister dans des zones-refuges. Si on suppose une arrivée depuis le sillon rhodanien, ces poulations seraient repoussées vers l'Est et/ou vers des territoires d'altitude. Dans le premier cas il serait intéressant de connaitre les chevaux italiens en vue de comparaisons. Dans le second cas, le gisement de Sault, et ses abords de plateaux calcaires, fournirait une bonne illustration de ce phénomène. A charge de cette hypothèse, 
on notera, outre la forte taille de notre sujet, un certain archaïsme révélé par l'indice

protoconique (cf. supra). hypothèses puissent fonctionner ensemble, par des processus interactifs entre une écoclimatologie (adaptation) et une dynamique de peuplement (isolat), pouvant justifier de la présence d'un cheval archaïque à la fin du Pléistocène en Provence4. l'abondance de cette espèce dans la zone péri-méditerranéenne en France (Provence, Languedoc) au cours du temps. Il paraît clair que la fin des temps glaciaires voit la disparition progressive des populations de chevaux, subsistant à l'état relictuel jusque vers 8000 ans, ainsi que dans les régions limitrophes. La fréquence des restes de chevaux dans les gisements cités est particulièrement révélatrice, à moins qu'il ne s'agisse d'une sélection anthropique liée à une chasse moins active dans les phases les plus récentes? (Ducos 1960).

Cette période est marquée par un réchauffement général accompagné d'un accroissement du couvert végétal et d'une diversification des groupes culturels de l'Epipaléolithique-Mésolithique régional. La forte réduction des zones herbacées marque la disparition du Cheval, animal d'espaces ouverts par excellence, pendant presque 3000 ans. Sa réapparition restera timide et ponctuelle à la fin du Néolithique, et pose le problème de la survivance de groupes dans les zones-refuges (localisation non connue) ou de sa domestication précoce dans nos régions? Il ne deviendra présent de manière régulière seulement à partir de l'Age du Fer sous forme domestique (origine est-européenne), mais le souvenir des derniers chevaux sauvages en Provence sera alors depuis longtemps oublié.

\section{BIBLIOGRAPHIE}

AUJARD-CATOT R., CREGUT E., GAGNIERE S., GRUNEISEN A., MOURER-CHAUVIRE C. 1979- Un squelette complet de renne Rangifer tarandus (Mammalia artiodactyla) dans le Pléistocène supérieur du remplissage karstique de l'aven des Planes (Monieux, Vaucluse, France). Nouvelles Archives du Muséum d'Histoire Naturelle de Lyon, 17 (suppl.), 1979, p. 15-20.

BAZILE F., GUILLERAULT P., MONNET C. 1986-1987- Le gisement épipaléolithique du Plaisir, Beauvoisin, Gard. Etude Quaternaire Languedocienne, 5, 1986-87, p. 21-34.

BON M., PICCOLI G., SALA B. 1999- I giacimenti quaterni di Vertebrati fossili nell'Italia nordorientale. Mem. Scienze Geologiche, Padova, 43, 1991, p. 185-231.

BONIFAY M.-F. 1968- La faune de l'abri Cornille (Istres, Bouches-du-Rhône). In : CNRS éds.- La Préhistoire : Problèmes et tendances, Paris, 1968, p. 47-57.

BONIFAY M.-F. 1977 - Essai sur l'histoire des faunes préhistoriques de Provence et du Languedoc. Bulletin de la Société d'Etude des Sciences Naturelles de Nîmes, 55, 1977, p. 23-39.

PALEO, 13 | 2001 
BONIFAY M.-F. 1978- Le gisement de la Baume de Valorgues à Saint-Quentin-la-Poterie (Gard) Paléolithique supérieur final et Epipaléolithique. 2- La Grande faune. Gallia Préhistoire, 21, 1978, p. 123-142.

BONIFAY M.-F., LECOURTOIS-DUCGONINAZ A. 1976- La faune des niveaux inférieurs de l'abri Cornille à Istres (Bouches-du-Rhône). Bulletin de la Société Préhistorique Française, Congrès préhistorique de France, 1974, XXe session, p. 228-247.

BROCHIER J.-E. 1977- Evolution des climats et des paysages vauclusiens au cours du Würmien récent et du Postglaciaire. Paléoécologie de l'homme fossile, Paris, CNRS, 1977, 180 p.

BRUGAL J.-P. 1981- Les faunes de grands mammifères du Pléistocène terminal en LanguedocOriental. Etude Quaternaire Languedocienne, nº sp. exc. AFEC, 1981, p. 21-28.

BRUGAL J.-P. 1982- Une faune du Tardiglaciaire dans l'aven Bouet (Les Matelles, Hérault). Etude Quaternaire Languedocienne, 2, 1982, p. 4-5.

BRUGAL J.-P. 1983- Apport à la chronoclimatologie du Würm récent dans les Alpes du Sud : la faune à Mégacéros du Pignon (Aspres-sur-Buëch, Hautes-Alpes). Préhistoire Anthropologie Méditerranéennes, 2, 1983, p. 29-41.

BUISSON-CATIL J., GRENET M., GASSIN B., HELMER D., JORDA M., MAGNIN F. 1991- La station de plein-air néolithique du Frigouras (Peipin, Alpes de Haute-Provence). Bulletin Archéologique de Provence, 20, 1991, p. 61-101.

BUISSON-CATIL J. 1993- L'aven des Fourches I (Sault, Vaucluse, France) : gisement de l'Age du Bronze final. In : Actes des Rencontres Néolithique et Protohistoire (ARENERA), 8, 1992-93. Lyon : Université Lumière-Lyon ; Valence : Centre d'Archéologie Préhistorique, 1992-93, p. 93-104.

BUISSON-CATIL J, SAUZADE G. 1995- Préhistoire des cavités karstiques des monts et plateaux de Vaucluse. In : Gaubert et al.- Les arcanes de Vaucluse. Hydrologie et spéléologie des territoires alimentant en eau la Fontaine de Vaucluse. Association de Recherches et d'Etudes Hydrologiques du Plateau d'Albion éd., t. 2, 1995, p. 126-143.

BUISSON-CATIL J. 1996- L'aven des Fourches I en Provence. Préhistoire et Protohistoire. Archéologia, n 325, juillet-août 1996, p. 52-57.

BUISSON-CATIL J., SAUZADE G., COUTURAUD A., HASLER A., PUIG J.-M., THIEBAULT S. 1996-1997Le gisement Bronze final de l'aven des Fourches I à Sault : contribution à l'étude fonctionnelle des cavités karstiques des plateaux de Vaucluse. Documents d'Archéologie Méridionale, 19-20, p. 7-31.

CALOI L., PALOMBO M.-R. 1994a- Le faune a grandi mammiferi del Pleistocene superiore dell'Italia centrale : biostratigraphia e paleoambiente. Boll. Serv. Geol. Italia, 111 (1992), 1994, p. 77-104.

CALOI L, PALOMBO M.-R. 1994b- Le principali faune a grandi mammiferi del Pleistocene superiore dell'Italia centrale. Studi Geologici Camerti, vol. sp. "Biostratigraphia dell'Italia centrale", 1994, p. 515-524.

COURTIN J. 1974- Le Néolithique de la Provence. Mémoires de la Société Préhistorique Française, 11, 1974, éd. Klincksieck, 359 p.

COURTIN J. 1978- Les animaux domestiques du Néolithique provençal : la faune sauvage et les débuts de l'élevage dans le Sud-Est de la France. Bulletin du Muséum National d'Histoire Naturelle de Marseille, 38, 1978, p. 187-194. 
COUTURAU A. 1995- La Fontaine de Vaucluse, émergence karstique. In : Gaubert et al.- Les arcanes de Vaucluse. Hydrologie et spéléologie des territoires alimentant en eau la Fontaine de Vaucluse. Association de Recherches et d'Etudes Hydrologiques du Plateau d'Albion éd., t. é, 1995, p. 103-117.

DEROSIER P. 1981- Contribution à l'étude hydrogéologique du cours supérieur de la Nesque. D.E.A., Université de Montpellier, 1981, 91 p.

DESBROSSE R. 1976- Les civilisations du Paléolithique supérieur dans le Jura méridional et dans les Alpes du Nord. In : H. de Lumley dir.- La Préhistoire Française, Paris, CNRS, 1976, t. I-2, p. 1196-1213.

DUCOS P. 1957- Etude de la faune du gisement de Roucadour (Lot). Bulletin du Musée d'Anthropologie Préhistorique de Monaco, 4, 1957, p. 165-188.

DUCOS P. 1960- Note sur le cheval néolithique. L'Anthropologie, 64, 1-2, 1960, p. 67-72.

GAUBERT G., LE FAHLER B., GILTZINGER P., PERRET J.-F., PUIG J.-M., SANNA J. 1990- Les cavernes d'Albion. Hydrologie et spéléologie des territoires alimentant en eau la Fontaine de Vaucluse. Association de Recherches et d'Etudes Hydrologiques du Plateau d'Albion éd, t. 1, 1990, 210 p.

GAUBERT G. 1993- Spéléologie des Hauts plateaux de Vaucluse et d'Albion. In : Pays de Sault et d'Albion. Hautes vallées de la Nesque et du Toulourenc et plateau d'Albion. Histoire, sites et monuments. Les Alpes de Lumière, 113, 1993, p. 14-17.

GAUBERT G., LE FAHLER B., BALLAND F., BEVENGUT P., BUISSON-CATIL J., COUTURAUD A., PUIG J.-M., SANNA J., SAUZADE G. 1995- Les arcanes de Vaucluse. Hydrologie et spéléologie des territoires alimentant en eau la Fontaine de Vaucluse. Association de Recherches et d'Etudes Hydrologiques du Plateau d'Albion éd, t. 2, 1995, 338 p.

GUADELLI J.-L. 1991- Les chevaux de Solutré (Saône-et-Loire). Cahiers du Quaternaire, 16, 1991, p. 261-336.

HELMER D. 1979- Recherches sur l'économie alimentaire et l'origine des animaux domestiques d'après l'étude des mammifères post-paléolithiques (du Mésolithique à l'Age du Bronze) en Provence. Doctorat 3ème cycle, 1979, Université des Sciences et techniques du Languedoc, Montpellier.

HELMER D. 1982- Etude de la faune. In : G. Sauzade, B. Gely- La grotte sépulcrale du Plan-desVaches à Gémenos (Bouches-du Rhône). Bulletin du Muséum National d'Histoire Naturelle de Marseille, 42, 1982, p. 87.

HELMER D. 1991- Etude la faune. In : J.-L. Roudil, H. Saumade éds- La grotte de Combe Obscure (Lagorce, Ardèche), 1991, p. 125-148.

HELMER D. 1992- La domestication des animaux par les hommes préhistoriques. Paris, éd. Masson, 1992, $184 \mathrm{p}$.

JORDA M. 1988- Du Pléniglaciaire supérieur au Postglaciaire : le concept de Tardiglaciaire européen et son évolution récente. Géologie Alpine, Mémoire hors série, 14, 1988, p. 241-249.

JORDA M. 1993- Les monts de Vaucluse et le plateau d'Albion. Paysages, géomorphologie, évolution quaternaire. In : Pays de Sault et d'Albion. Hautes vallées de la Nesque et du Toulourenc et plateau d'Albion. Histoire, sites et monuments. Les Alpes de Lumière, 113, 1993, p. 8-13.

LECOURTOIS-DUC GONINAZ A. 1984- Les habitats épipaléolithiques du Mourre-Poussiou à Fossur-Mer (Bouches-du-Rhône). II. La faune. Gallia Préhistoire, 27, 1, 1984, p. 81-95. 
LEVINE M.-A. 1982- The use of crown height measurements and eruption-wear sequences to age horse teeth. In: B. Wilson, C. Grigson, S. Payne eds- Ageing and Sexing animal bones from archaeological sites. BAR British series, Oxford, 109, 1982, p. 223-250.

MASSETI M., RUSTIONI M., SALA B. 1995- Large-sized italian ungulates at the Late PleistoceneHolocene transition: an overview. PADUSA Quaderni, Atti del $1^{\circ}$ Conv. Naz. di archeozoologia, 1 , 1995, p. 89-96.

MAGNY M. 1995- Une histoire du climat. Des derniers mammouths au siècle de l'automobile. Paris, 1995, éd. Errance, 176 p.

ONORATINI G. 1982- Préhistoire, sédiments, climats du Würm III à l'Holocène dans le Sud-Est de la France. Thèse d'Etat, 1982, Fac. Sc. et Tech., Saint-Jérôme, Marseille, 2 vol.

PACCARD M., BOUVILLE C. 1993- coll.- La grotte d'Unang. Malemort-du-Comtat. Documents d'Archéologie Vauclusienne, 4, 1993, 205 p. 12 pl.

PAREIN R., LANGUILLE A. 1981- La Haute Provence souterraine. Ed. compte d'auteurs, 1981, 422 p. POPLIN F., POULAIN T., MENIEL P., VIGNE J.-D., GEDDES D., HELMER D. 1986- Les débuts de l'élevage en France. In : J.-P. Demoule, J. Guilaine (dir.)- Le Néolithique de la France. Hommage à G. Bailloud. Paris, Picard éd., 1986, p. 37-51.

POULAIN T. 1971- Le camp mésolithique de Gramari à Méthamis (Vaucluse). III- Etude de la faune. Gallia Préhistoire, 14, 1, 1971, p. 121-131.

PUIG J.-M. 1987- Le système karstique de la Fontaine de Vaucluse. Thèse 3ème cycle, Université d'Avignon (Doc. BRGM, 180, 1990), 208 p., 69 fig., 27 tbl.

PUIG J.-M. 1990- L'impluvium de la Fontaine de Vaucluse, morphologie, géologie, hydrologie. In : Gaubert et al.- Les cavernes d'Albion. Hydrologie et spéléologie des territoires alimentant en eau la Fontaine de Vaucluse. Association de Recherches et d'Etudes Hydrologiques du Plateau d'Albion éd., t. 1, 1990, p. 15-32.

RIVIER F. 1960- Etude géologique des monts de Vaucluse. Rapport COPEFA, Permis de Die, 1960.

SCHVOERER M., BORDIER C., EVIN J., DELIBRIAS G. 1977- Eléments pour la chronologie absolue de la fin des temps glaciaires en Europe. Recensement et présentation des datations se rapportant à des sites français. In : La fin des temps glaciaires en Europe. Chronostratigraphie et écologie des cultures du Paléolithique final. Coll. int. CNRS, bordeaux, éd. C.R.I.A.A., 1977, p. 1-14.

VILLEGER M. 1984- Evolution du panneau de couverture nord-provençal (Mont Ventoux, Luberon, Moyenne Durance), France. Thèse 3ème cycle, Universite Paris-sud Orsay, 1984, 174 p., 50 fig.

WEYDERT P. 1990- La morphologie karstique de l'extrémité orientale des monts de Vaucluse. In : Gaubert et al.- Les cavernes d'Albion. Hydrologie et spéléologie des territoires alimentant en eau la Fontaine de Vaucluse. Association de Recherches et d'Etudes Hydrologiques du Plateau d'Albion éd., t. 1, 1990, p. 35-69.

\section{ANNEXES}




\section{Gisements cités dans le texte présentant des restes d' Equus caballus}

\section{Provence}

\section{1- Tardiglaciaire}

Vaucluse : Soubeyras, aven des Planes, Chinchon, Unang, Roquefure

Bouches-du-Rhône : Adaouste, Cornille

Var : Rainaude, Bouverie, Baume Goulon, Vieux-Mounoï

\section{2- Postglaciaire}

Vaucluse : Gramari, La Balance, Unang

Bouches-du-Rhône : Les Calades, Mourre-Poussiou, Plan-des-Vaches

Var : Eglise supérieure

Alpes de Haute-Provence : Frigouras, Saint-Benoît

Alpes-Maritimes : Barriéra

\section{Languedoc-Causse}

\section{1- Tardiglaciare}

Gard : Salpétrière, Valorgues, Oullins

Hérault : Aven Bouet

\section{2- Postglaciare}

Gard : St-Côme-de-Maruéjols, Le Plaisir

Ardèche : Combe Obscure

Hérault : Le Lébous, Gravas

Lot : Roucadour, Reilhac, abri Pagès

\section{Dauphiné-Alpes}

\section{1- Tardiglaciaire}

Isère : Calvaire

Drôme : Tai, Campalou

\section{NOTES}

1. Analyse effectuée par Y. Quinif. Centre d'Etudes et de Recherches Appliquées au Karst (CERAK). Faculté Polytechnique de Mons, Belgique. 
2. Exprimées en années, mois (soit 1,3 = 1 an et 3 mois)

3. Il serait cependant encore présent dans les niveaux Bronze ancien de La Rouyère au Beaucet dans le Vaucluse ( $\mathrm{n}=2$; Helmer, inédit)

4. Concernant un groupe différent, les Mégacéridés, on relèvera la présence d'une forme de petite taille de M. giganteus ssp., datée d'environ 19000 B.P., dans un environnement de vallées d'altitude (700-800m) des hautes-Alpes, pouvant également résulter d'un mécanisme d'isolement (Brugal 1993).

\section{RÉSUMÉS}

La découverte de restes de chevaux dans un contexte d'aven permet de présenter un ensemble d'études sur les conditions de dépôt et la taphonomie ainsi que de préciser l'attribution taxonimique du matériel. Celui-ci, daté par ${ }^{14} \mathrm{C}$ de $12500 \pm 300$ ans B.P., serait proche de la forme ancienne germanicus; elle révèle ainsi des conditions biogéographiques et/ou écologiques particulières pour la région concernée. Cette découverte permet de faire le point sur l'existence et l'abondance de cette espèce dans la zone péri-méditerranéenne (Provence, Languedoc) au Tardiglaciaire et au Postglaciaire.

We report the recent find from a sinkhole of horse remains which allows us to precise the geological context as well as the taphonomy and taxinomy of the fossil material. The bones are $14 \mathrm{C}$ dated of $12500+/-300$ years B.P. and this species is closed to an ancient form named germanicus. Its presence evidences some specific biogeographical and/or ecological conditions for this region. This discovery draws up an inventory of wild horses (presence and abundance) in the peri-mediterranean areas (Provence, Languedoc) during Tardiglacial and Postglacial times.

\section{INDEX}

Keywords : Equids, karst, Languedoc, Postglacial, Provence, Taphonomy, Tardiglacial, Taxinomy Mots-clés : Equidés, karst, Languedoc, Postglaciaire, Provence, taphonomie, Tardiglaciaire, taxinomie

\section{AUTEURS}

\section{JEAN-PHILIP BRUGAL}

UMR 6636 - ESEP - Maison Méditerranéenne des Sciences de l'Homme, BP647, F-13094 Aix-enProvence, brugal@mmsh.univ-aix.fr

\section{JACQUES BUISSON-CATIL}

Service Régional de l'Archéologie PACA, 23, blv. Du Roi René, F-13617 Aix-en-Provence et UMR 6636 - ESEP - Maison Méditerranéennes des Sciences de l'Homme 


\section{DANIEL HELMER}

UPR 7537 du CNRS : Origines et développement de la sédentarisation au Proche-Orient. Institut de Préhistoire orientale. Jalès, 07460 Berrias. 